\title{
Alimentação de peixes: proposta para análise de itens registrados em conteúdos gástricos
}

\author{
Sirlei Terezinha Bennemann ${ }^{1}$, Lilian Casatti ${ }^{2}$ \& Deise Cristiane de Oliveira ${ }^{1}$
}

Biota Neotropica v6 (n2) - http://www.biotaneotropica.org.br/v6n2/pt/abstract?article+bn01206022006

Recebido em 27/07/05.

Versão revisada recebida em 08/04/06.

Publicado em 01/05/06.

\begin{abstract}
${ }^{1}$ Departamento de Biologia Animal e Vegetal, Centro de Ciências Biológicas (CCB), Universidade Estadual de Londrina (www.uel.br), Rodovia Celso Garcia Cid (PR 445), km 380, 86051-990 Londrina, Paraná, Brasil (e-mail: sirlei@uel.br, deisecristiane@yahoo.com.br)

${ }^{2}$ UNESP - Universidade Estadual Paulista, Laboratório de Ictiologia, Departamento de Zoologia e Botânica, IBILCE (www.ibilce.unesp.br), Rua Cristóvão Colombo, 2265, 15054-000 São José do Rio Preto, São Paulo, Brasil (e-mail: $\underline{\text { lcasatti@ibilce.unesp.br) }}$
\end{abstract}

\begin{abstract}
Sirlei Terezinha Bennemann, S. T.; Casatti, L. and Oliveira, D.C. Fish feeding: a proposal for analysis of the stomach content. Biota Neotrop. May/Sep 2006 vol. 6, no. 2, http://www.biotaneotropica.org.br/v6n2/pt/abstract?article+bn01206022006. ISSN 1676-0603

This study aimed to test a simplified protocol for feeding fish analysis. For this, the food items found in the gastric contents of six freshwater fish species (Astyanax paranae, Phalloceros caudimaculatus, Loricariichthys platymetopon, Moenkhausia intermedia, Schizodon intermedius, and Plagioscion squamosissimus) were quantified by weight (\%) and dominance (\%). Using the quantification by weight for each food item, the IAi (Feeding Index) was calculated and further compared with the dominance (D) using the Morisita-Horn similarity coefficient. The food items with higher values of IAi were also those with the largest dominance values. The general similarity between IAi and dominance (D) was high, varying of $87 \%$ (A. paranae) to 99\% (P. caudimaculatus, L. platymetopon, S. intermedius, and P. squamosissimus), showing that dominance can replace composed indexes calculation. The graphic representation of weight and dominance against the frequency of occurrence showed that both are adequate to identify the most important food items, revealing that dominance may alternatively be used instead of prey items weighting, simplifying the data collection and analysis. When the objective of the trophic study is to identify the food items that stand out in quantitative terms or investigate specialization/generalization tendencies, we recommend the use of the dominance in combination to graphic methods.
\end{abstract}

Key words: trophic ecology, dominance, weight, frequency of occurrence, feeding index (IAi), methods

\section{Resumo}

Sirlei Terezinha Bennemann, S. T.; Casatti, L. and Oliveira, D.C. Alimentação de peixes: proposta para análise de itens registrados em conteúdos gástricos. Biota Neotrop. May/Sep 2006 vol. 6, no. 2,http://www.biotaneotropica.org.br/ v6n2/pt/abstract?article+bn01206022006. ISSN 1676-0603

Este estudo teve o objetivo de testar um protocolo simplificado para análise da alimentação de peixes. Para tal, os itens alimentares encontrados nos conteúdos gástricos de seis espécies de peixes de água doce (Astyanax paranae, Phalloceros caudimaculatus, Loricariichthys platymetopon, Moenkhausia intermedia, Schizodon intermedius e Plagioscion squamosissimus) foram quantificados através do peso (\%) e da dominância (\%). Utilizando a quantificação de cada item através do peso, foi calculado o IAi (índice alimentar), posteriormente comparado com a dominância (D) através do coeficiente de similaridade de Morisita-Horn. Os itens alimentares com maiores valores de IAi também foram aqueles com os maiores valores de dominância, sendo que em uma análise geral a semelhança entre ambos foi alta, variando de $87 \%$ (A. paranae) a 99\% (P. caudimaculatus, L. platymetopon, $S$. intermedius e $P$. squamosissimus), mostrando que a dominância pode substituir os cálculos de índices compostos. Através da representação gráfica do peso e da dominância em função da freqüência de ocorrência, fica claro que ambos são adequados para identificar os itens alimentares mais importantes e que a dominância pode ser utilizada como alternativa à pesagem das presas, simplificando a etapa de coleta e análise de dados. Assim, se o objetivo do estudo trófico for identificar o item alimentar que se destaca em termos quantitativos ou investigar tendências de especialização/ generalização trófica, recomendamos o emprego da dominância em associação com métodos gráficos.

Palavras-chave: ecologia trófica, dominância, peso, freqüência de ocorrência, índice alimentar (IAi), métodos 


\section{Introdução}

O conjunto mais acessível de informações sobre alimentação de peixes é obtido de forma indireta, através do tradicional exame dos conteúdos gástricos (Windell \& Bowen 1978). Observações diretas durante mergulho permitem coletar dados enriquecedores, porém, nem sempre essa abordagem pode ser aplicada com facilidade em alguns ambientes, especialmente os de água doce (veja Uieda \& Castro 1999 e Sabino 1999 para discussões sobre métodos de coleta e observação de peixes de água doce no Brasil), além de ser impreciso inferir a dieta de uma espécie apenas por meio de observações diretas, em razão das pequenas dimensões de muitos predadores e presas, tornando-se necessária a associação desta prática com a análise dos conteúdos gástricos.

As publicações de Hynes (1950), Hyslop (1980) e Bowen (1992) estão entre as mais citadas revisões sobre métodos utilizados no estudo da alimentação de peixes. Esses autores apontam dificuldades na padronização de tais métodos, o que se reflete no grande número de estratégias para quantificação de itens alimentares (vide Hahn \& Delariva 2003, para uma recente revisão). Alguns desses métodos foram combinados em índices alimentares com o objetivo de destacar quais as presas que mais contribuem para a alimentação dos predadores (Rosecchi \& Nouaze 1987). Atualmente há pelo menos dez diferentes versões disponíveis de índices compostos, apesar da nítida tendência em suprimir-se seu uso (Hahn \& Delariva 2003). Quando tais índices são empregados, os que se destacam são o Índice Alimentar (IAi, Kawakami \& Vazzoler 1980) nos estudos publicados em periódicos nacionais e o Índice de Importância Relativa (IRI, Pinkas et al. 1971), nos internacionais (Hahn \& Delariva 2003). O IAi, tal como proposto originalmente, é baseado na freqüência de ocorrência e no volume de cada item, mas alguns autores o utilizaram com modificações, substituindo o volume por peso (Hahn et al. 1997, Oliveira et al. 2004) ou o volume por pontos (Fugi et al. 1996, Vitule \& Aranha 2002). No caso do IRI, são combinados os percentuais da freqüência de ocorrência, do peso e do número de componentes de cada item.
Na busca de métodos mais eficientes para sintetizar as informações contidas nos conteúdos gástricos, Costello (1990) propôs um método gráfico capaz de discriminar se a presa é rara ou dominante. Para o emprego deste método é necessário, além da freqüência de ocorrência, o cálculo da abundância percentual de cada item, quantificada por peso ou volume. Amundsen et al. (1996) propuseram modificações ao método gráfico de Costello (1990) no intuito de explorar a importância relativa de cada presa, a estratégia alimentar e os componentes individuais de amplitude de nicho trófico. Contudo, tais modificações pressupõem um número adequado de exemplares (geralmente maior que 30) e estômagos relativamente repletos de conteúdo.

$\mathrm{O}$ maior desafio, no entanto, ainda se refere à quantificação de itens de diferentes tamanhos, consistência e estado de digestão, procurando não subestimar a importância de itens numerosos e não superestimar a importância de itens raros, o que geralmente ocorre em análises baseadas somente em ocorrência (Aranha, 1993).

Em geral, estudos envolvendo ecologia trófica buscam identificar os hábitos alimentares através da análise dos principais itens consumidos pelas espécies. Especialmente no que diz respeito à ictiofauna de água doce Neotropical, onde são estimadas até oito mil espécies (Schaefer 1998), ainda desconhecemos boa parte da dieta e seus hábitos alimentares e, em muitas ocasiões, a comparação entre informações publicadas mostra-se limitada em função da diversidade e falta de padronização dos métodos de análise utilizados. Em vista de tais restrições, nosso objetivo foi testar um protocolo simplificado para análise da alimentação de peixes.

\section{Material e Métodos}

Seis espécies de peixes de águas continentais sulamericanas foram utilizadas para testar o protocolo em questão e foram selecionadas em função de representarem distintos grupos tróficos, hábitat e tamanho corporal (Tabela 1). Todos os exemplares examinados estão depositados na coleção ictiológica do Museu de Zoologia da Universidade Estadual de Londrina (MZUEL), Londrina, Brasil. 

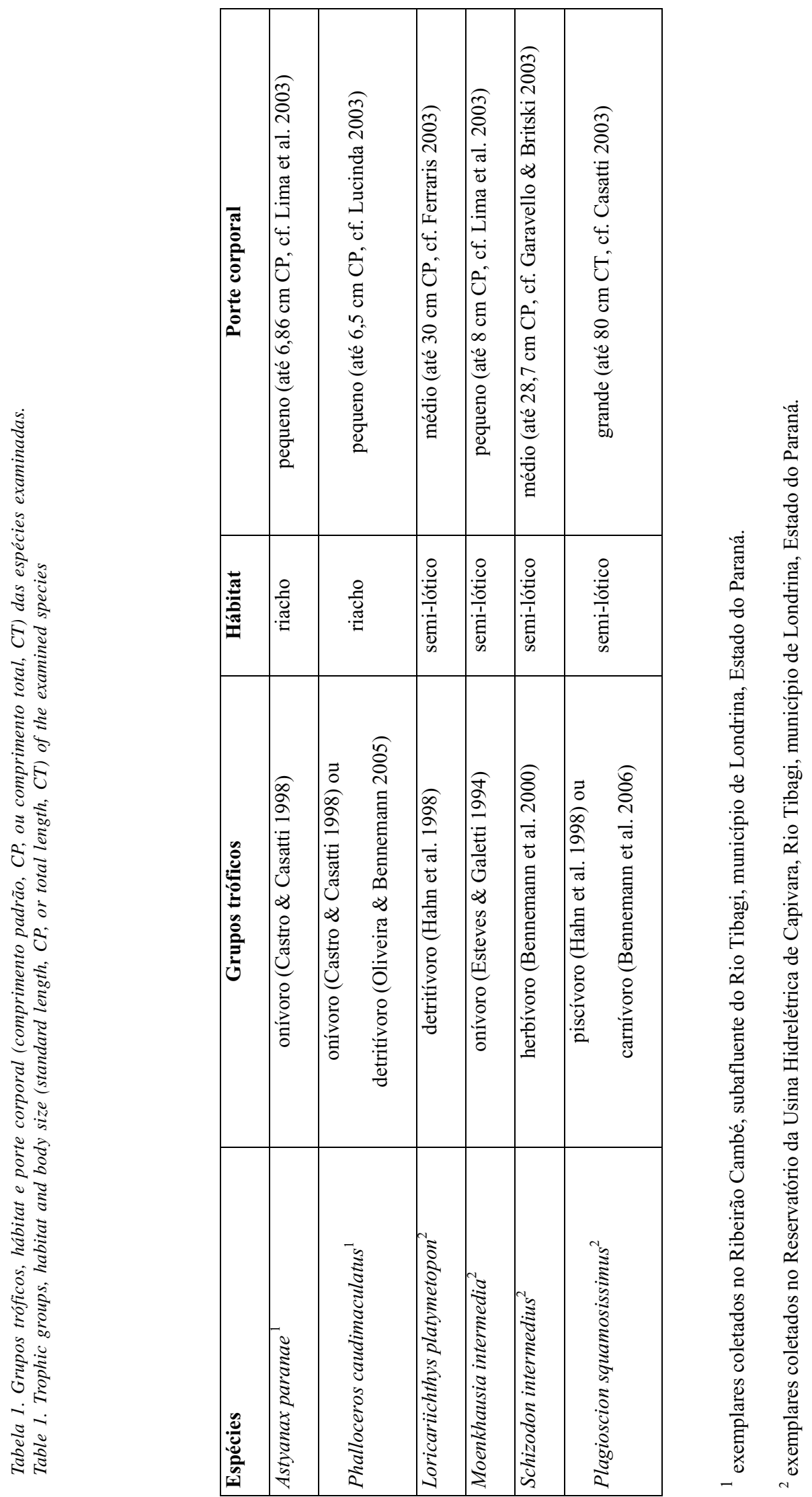

http://www.biotaneotropica.org.br 
Cada item alimentar registrado nos conteúdos gástricos foi quantificado através do peso (método gravimétrico, Hyslop 1980), sendo expresso como a porcentagem do peso total de todos os itens encontrados num dado estômago, e da dominância (Frost \& Went 1940 apud Hynes 1950), esta dada como a porcentagem do número de vezes em que o item ocupa a maior parte do conteúdo de cada estômago em função do número total de exemplares analisados.

Para determinar a importância de cada item foi utilizado o método gráfico de Costello (1990), onde peso (\%) e dominância (\%) foram representados no eixo y e a freqüência de ocorrência (\%) no eixo x. A inclusão da dominância no método gráfico de Costello (1990) é uma inovação proposta no presente estudo. Para cada item alimentar foi também calculado o Índice Alimentar (IAi), seguindo proposta de Kawakami \& Vazzoler (1980), porém, substituindo-se o volume pelo peso de cada item (Hahn et al. 1997, Oliveira et al. 2004). Para cada espécie foi avaliada a semelhança entre os valores de IAi e D, através do cálculo do índice de

Tabela 2. Amplitude de comprimento padrão $(\mathrm{CP}, \mathrm{cm})$, número de exemplares analisados (n), ocorrência de cada item (oc), freqüência de ocorrência (\% FO), peso ( $p$, em gramas), porcentagem em peso de cada item (\% $\mathrm{P}$ ), número de estômagos em que determinado item foi dominante (d), dominância (\% D) e Índice Alimentar (\% IAi) de Astyanax paranae $e$ Phalloceros caudimaculatus. similaridade de Morisita-Horn, um coeficiente adequado para analisar proporções e que recebe pouca influência do tamanho das amostras (Krebs 1999).

\section{Resultados}

Os itens alimentares com maiores valores de IAi foram também aqueles com os maiores valores de D (Tabelas 2 e 3). Além disso, os valores de IAi e D para alguns itens em particular foram muito semelhantes, notavelmente para detritos na dieta de Phalloceros caudimaculatus (Tabela 2) e Loricariichthys platymetopon (Tabela 3) e para Caridea na dieta de Plagioscion squamosissimus (Tabela 3). Em termos gerais, a similaridade entre IAi e D foi alta, sendo de 87\% para Astyanax paranae, 92\% para Moenkhausia intermedia e 99\% para as demais espécies ( $P$. caudimaculatus, L. platymetopon, Schizodon intermedius e P. squamosissimus).

Table 2. Range of standard length (CP, $\mathrm{cm})$, number of analyzed specimens ( $n$ ), occurrence of each item (oc), frequency of occurrence (\% FO), weigh ( $p$, in grams), percentage in weight of each item $(\% P)$, number of stomachs with certain item dominant $(d)$, dominance (\% D) and Alimentary Index (\% IAi) of Astyanax scabripinnis and Phalloceros caudimaculatus.

\begin{tabular}{|c|c|c|c|c|c|c|c|}
\hline \multicolumn{8}{|c|}{ Astyanax paranae $(\triangle \mathrm{CP}=2,4-8,0, \mathrm{n}=102)$} \\
\hline Itens alimentares: & oc & $\%$ FO & $\mathbf{p}$ & $\% \mathbf{P}$ & d & $\% \mathrm{D}$ & \% IAi \\
\hline 1) Gramíneas & 16 & 15,7 & 0,923 & 18,0 & 10 & 9,8 & 9,6 \\
\hline 2) Restos vegetais & 66 & 64,7 & 1,443 & 28,2 & 36 & 35,2 & 62,1 \\
\hline 3) Coleoptera & 17 & 16,7 & 0,335 & 6,6 & 7 & 6,9 & 3,7 \\
\hline 4) Ephemeroptera & 4 & 3,9 & 0,321 & 6,3 & 3 & 2,9 & 0,8 \\
\hline 5) Hemiptera & 3 & 2,9 & 0,137 & 2,7 & 1 & 1,0 & 0,3 \\
\hline 6) Hymenoptera & 23 & 22,5 & 0,459 & 9,0 & 13 & 12,8 & 6,9 \\
\hline 7) Lepidoptera & 2 & 2,0 & 0,162 & 3,2 & 2 & 2,0 & 0,2 \\
\hline 8) Odonata & 1 & 1,0 & 0,003 & 0,1 & - & - & $<0,1$ \\
\hline 9) Chironomidae & 2 & 2,0 & 0,008 & 0,2 & 1 & 1,0 & $<0,1$ \\
\hline 10) Culicidae & 3 & 2,9 & 0,051 & 1,0 & 1 & 1,0 & 0,1 \\
\hline 11) Outros Diptera & 10 & 9,8 & 0,197 & 3,9 & 3 & 2,9 & 1,3 \\
\hline 12) Restos de insetos & 35 & 34,3 & 0,590 & 11,5 & 19 & 18,6 & 13,4 \\
\hline 13) Isopoda & 1 & 1,0 & 0,170 & 3,3 & 3 & 2,9 & 0,1 \\
\hline 14) Peixes & 1 & 1,0 & 0,014 & 0,3 & - & - & $<0,1$ \\
\hline 15) Diversos táxons & 3 & 2,9 & 0,071 & 1,4 & 1 & 1,0 & 0,1 \\
\hline 16) Testacealobosia & 1 & 1,0 & 0,005 & 0,1 & - & - & $<0,1$ \\
\hline 17) Detritos & 11 & 10,8 & 0,098 & 1,9 & 1 & 1,0 & 0,7 \\
\hline 18) Sedimentos & 7 & 6,9 & 0,130 & 2,5 & 1 & 1,0 & 0,6 \\
\hline Total & 206 & - & 5,117 & 100 & 102 & 100 & 100 \\
\hline \multicolumn{8}{|c|}{ Phalloceros caudimaculatus $(\Delta C P=2,1-3,5, n=56)$} \\
\hline 1) Restos vegetais & 3 & 5,4 & 0,0067 & 0,7 & - & - & $<0,1$ \\
\hline 2) Coleoptera & 2 & 3,6 & 0,0107 & 1,1 & 1 & 1,8 & $<0,1$ \\
\hline 3) Hymenoptera & 2 & 3,6 & 0,0216 & 2,2 & - & - & 0,1 \\
\hline 4) Ceratopogonidae & 1 & 1,8 & 0,0032 & 0,3 & - & - & $<0,1$ \\
\hline 5) Chironomidae & 10 & 17,9 & 0,0344 & 3,6 & 1 & 1,8 & 0,8 \\
\hline 6) Muscidae & 3 & 5,4 & 0,0145 & 1,5 & - & - & 0,1 \\
\hline 7) Outros Diptera & 4 & 7,1 & 0,0257 & 2,7 & 2 & 3,6 & 0,2 \\
\hline 8) Restos de insetos & 12 & 21,4 & 0,0357 & 3,7 & 2 & 3,6 & 1,0 \\
\hline 9) Diversos táxons & 1 & 1,8 & 0,0045 & 0,5 & 1 & 1,8 & $<0,1$ \\
\hline 10) Testacealobosia & 13 & 23,2 & 0,0119 & 1,2 & - & - & 0,3 \\
\hline 11) Detritos & 56 & 100,0 & 0,7748 & 80,6 & 49 & 87,4 & 97,2 \\
\hline 12) Sedimentos & 6 & 10,7 & 0,0175 & 1,8 & - & - & 0,2 \\
\hline Total & 113 & - & 0,9612 & 100 & 56 & 100 & 100 \\
\hline
\end{tabular}


Tabela 3. Amplitude de comprimento padrão (CP, cm), número de exemplares analisados (n), número de ocorrência de cada item (oc), freqüência de ocorrência (\% FO), peso ( $p$, em gramas), porcentagem em peso de cada item (\% P), número de estômagos em que determinado item foi dominante (d), dominância (\% D) e Índice Alimentar (\% IAi) de Locariichthys platymetopon, Moenkhausia intermedia, Schizodon intermedius $e$ Plagioscion squamosissimus.

Table 3. Range of standard length (CP, cm), number of analyzed specimens ( $n$ ), occurrence of each item (oc), frequency of occurrence (\% $F O)$, weigh ( $p$, in grams), percentage in weight of each item (\%P), number of stomachs with certain item dominant (d), dominance (\% D) and Alimentary Index (\% IAi) of Locariichthys platymetopon, Moenkhausia intermedia, Schizodon intermedius e Plagioscion squamosissimus.

\begin{tabular}{|c|c|c|c|c|c|c|c|}
\hline \multicolumn{8}{|c|}{ Loricariichthys platymetopon $(\triangle \mathrm{CP}=16,3-30,5, \mathrm{n}=20)$} \\
\hline Itens alimentares: & oc & $\%$ FO & $\mathbf{p}$ & $\% \mathbf{P}$ & d & $\% \mathrm{D}$ & $\%$ IAi \\
\hline 1) Ostracoda & 15 & 75,0 & 0,244 & 8,0 & 1 & 5,0 & 6,7 \\
\hline 2) Cladocera & 3 & 15,0 & 0,055 & 1,8 & 1 & 5,0 & 1,3 \\
\hline 3) Copepoda & 1 & 5,0 & 0,051 & 1,7 & - & - & 0,3 \\
\hline 4) Chironomidae & 1 & 5,0 & 0,013 & 0,4 & - & - & 0,1 \\
\hline 5) Restos vegetais & 9 & 45,0 & 0,038 & 1,2 & - & - & 0,6 \\
\hline 6) Detritos & 19 & 95,0 & 2,613 & 85,3 & 18 & 90,0 & 90,8 \\
\hline 7) Sedimentos & 4 & 20,0 & 0,051 & 1,7 & - & - & 0,2 \\
\hline Total & 52 & - & 3,065 & 100 & 20 & 100 & 100 \\
\hline
\end{tabular}

Moenkhausia intermedia $(\triangle \mathrm{CP}=\mathbf{8 , 9}-\mathbf{9 , 5}, \mathrm{n}=\mathbf{2 2})$

\begin{tabular}{|l|c|c|c|c|c|c|c|}
\hline 1) Caridea & 11 & 50,0 & 0,368 & 23,8 & 8 & 36,4 & 22,6 \\
\hline 2) Cladocera & 18 & 81,8 & 0,744 & 46,0 & 11 & 50,0 & 71,6 \\
\hline 3) Trichoptera & 1 & 4,5 & 0,036 & 2,2 & - & - & 0,2 \\
\hline 4) Chironomidae & 2 & 9,0 & 0,055 & 3,4 & - & - & 0,6 \\
\hline 5) Restos de insetos & 1 & 4,5 & 0,008 & 0,5 & - & - & $<0,1$ \\
\hline 6) Restos vegetais & 3 & 13,6 & 0,117 & 7,2 & - & - & 1,9 \\
\hline 7) Quirela* & 3 & 13,6 & 0,154 & 9,5 & 3 & 13,6 & 2,5 \\
\hline 8) Detritos & 1 & 4,5 & 0,009 & 0,6 & - & - & $<0,1$ \\
\hline 9) Sedimentos & 1 & 4,5 & 0,110 & 6,8 & - & - & 0,6 \\
\hline Total & $\mathbf{4 1}$ & - & $\mathbf{1 , 6 1 9}$ & $\mathbf{1 0 0}$ & $\mathbf{2 2}$ & $\mathbf{1 0 0}$ & $\mathbf{1 0 0}$ \\
\hline
\end{tabular}

Schizodon intermedius $(\triangle \mathrm{CP}=12,4-31,5, \mathrm{n}=62)$

\begin{tabular}{|l|c|c|c|c|c|c|c|}
\hline 1) Algas filamentosas & 37 & 59,7 & 27,290 & 21,5 & 10 & 16,1 & 19,4 \\
\hline 2) Quirela* & 7 & 11,3 & 19,460 & 15,3 & 4 & 6,5 & 2,6 \\
\hline 3) Restos vegetais & 55 & 88,7 & 71,159 & 56,0 & 45 & 72,6 & 75,0 \\
\hline 4) Restos de insetos & 12 & 19,4 & 0,460 & 0,4 & - & - & 0,1 \\
\hline 5) Cladocera & 7 & 11,3 & 0,275 & 0,2 & - & - & $<0,1$ \\
\hline 6) Gastropoda & 1 & 1,6 & 0,020 & 0,0 & - & - & $<0,1$ \\
\hline 7) Porifera & 1 & 1,6 & 0,060 & 0,1 & - & - & $<0,1$ \\
\hline 8) Testacealobosia & 20 & 32,3 & 0,272 & 0,2 & - & - & 0,1 \\
\hline 9) Detritos & 20 & 32,3 & 6,535 & 5,1 & 3 & 4,8 & 2,5 \\
\hline 10) Sedimentos & 7 & 11,3 & 1,630 & 1,2 & - & - & 0,2 \\
\hline Total & $\mathbf{1 6 7}$ & - & $\mathbf{1 2 7 , 1 6 1}$ & $\mathbf{1 0 0}$ & $\mathbf{6 2}$ & $\mathbf{1 0 0}$ & $\mathbf{1 0 0}$ \\
\hline
\end{tabular}

Plagioscion squamosissimus $(\triangle \mathrm{CP}=15,8-28,9, \mathrm{n}=36)$

\begin{tabular}{|l|c|c|c|c|c|c|c|}
\hline 1) Restos vegetais & 5 & 13,9 & 0,670 & 1,4 & - & - & 0,3 \\
\hline 2) Odonata & 6 & 16,7 & 0,085 & 1,8 & 1 & 2,8 & 0,4 \\
\hline 3) Restos de insetos & 1 & 2,8 & 0,050 & 0,1 & - & - & $<0,1$ \\
\hline 4) Caridea & 33 & 91,7 & 23,336 & 49,7 & 23 & 63,9 & 66,8 \\
\hline 5) Peixes & 17 & 47,2 & 22,800 & 47,0 & 12 & 33,3 & 32,5 \\
\hline Total & $\mathbf{6 2}$ & - & $\mathbf{4 6 , 2 2 1}$ & $\mathbf{1 0 0}$ & $\mathbf{3 6}$ & $\mathbf{1 0 0}$ & $\mathbf{1 0 0}$ \\
\hline
\end{tabular}

* milho quebrado que é utilizado como ceva pelos pescadores da região 
Quando representados através do método gráfico de Costello (1990), peso e dominância indicam claramente os itens principais na alimentação de todas as espécies e grupos tróficos analisados, independentemente de
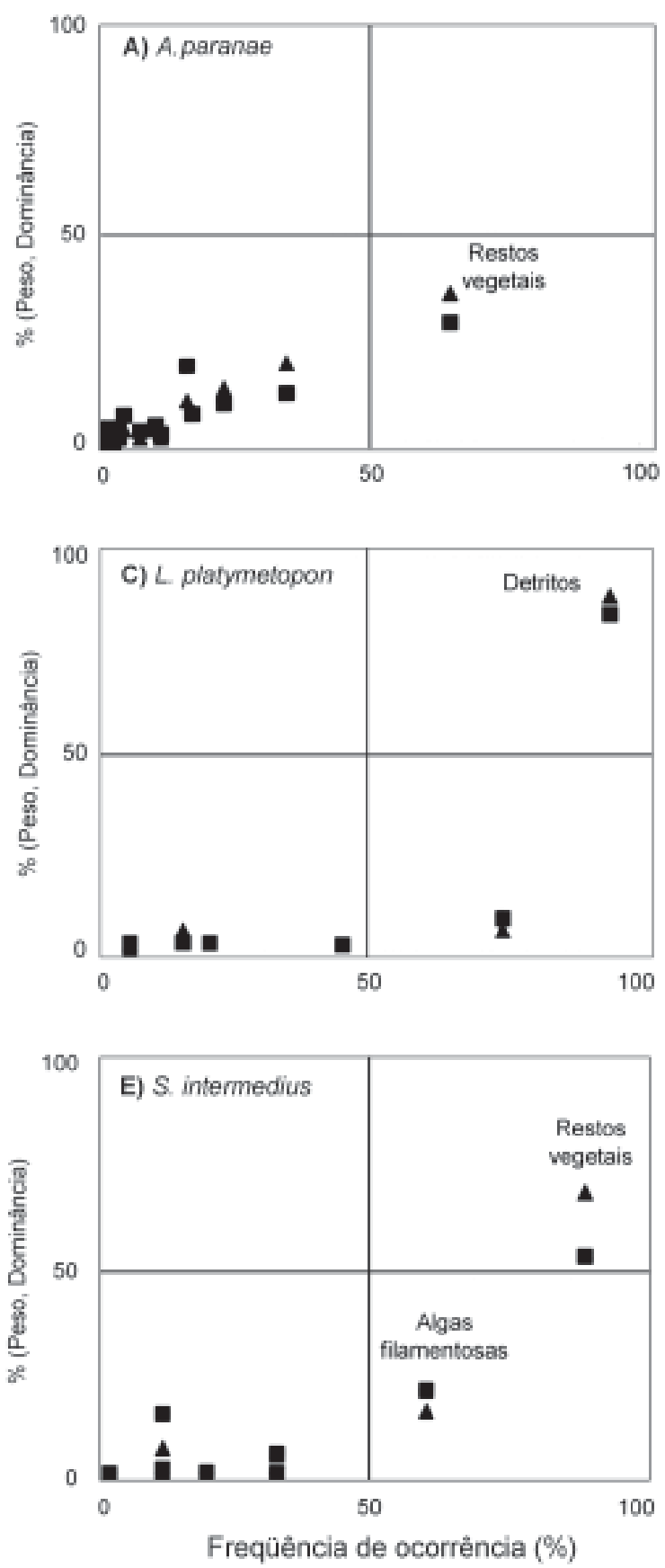

apresentarem tendência à especialização $(P$. caudimaculatus, $L$. platymetopon, S. intermedius e $P$. squamosissimus) ou à generalização alimentar (A. paranae, M. intermedia) (Figura 1).
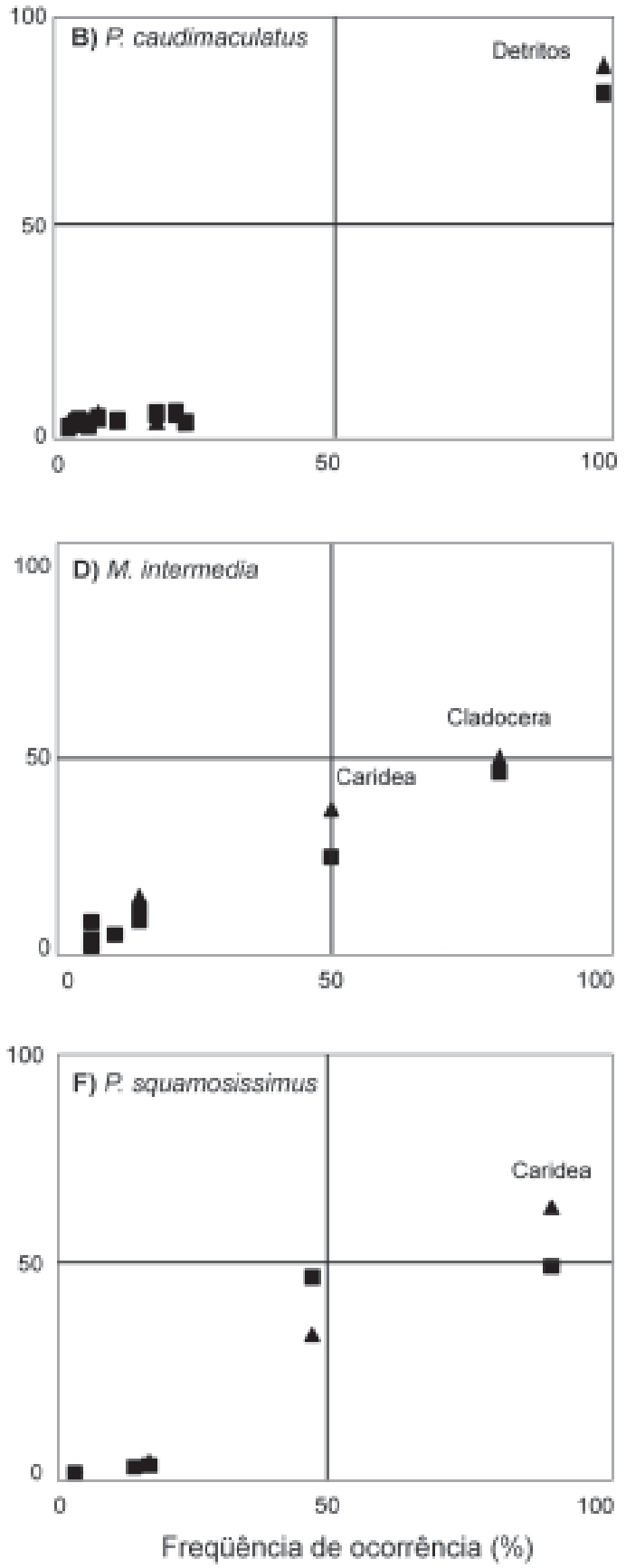

- Peso $\Delta$ Dominância

Figura 1. Representação gráfica do peso e da dominância (eixo y) em função da freqüência de ocorrência (eixo x) dos itens alimentares registrados nos conteúdos gástricos de (A) Astyanax paranae, (B) Phalloceros caudimaculatus, (C) Loricariichthys platymetopon, (D) Moenkhausia intermedia, (E) Schizodon intermedius $e(F)$ Plagioscion squamosissimus.

Figure 1. Graphic representation of the weight and dominance (axis y) in function of the frequency of occurrence (axis $x$ ) of the food items registered in the gastric contents of (A) Astyanax scabripinnis, (B) Phalloceros caudimaculatus, (C) Loricariichthys platymetopon, (D) Moenkhausia intermedia, (E) Schizodon intermedius and (F) Plagioscion squamosissimus. 


\section{Discussão}

Em estudos de ecologia trófica de peixes geralmente o objetivo é determinar qual o item de maior importância e, desta forma, obter informações para a descrição da dieta e hábito alimentar de uma espécie. Essa importância pode ser interpretada tanto em termos de ocorrência, volume, peso e/ou valor energético (Hyslop 1980) e cada um desses componentes pode ser investigado em função da pergunta ecológica que se pretende responder. Na maioria das vezes, a ocorrência e a quantidade (dada geralmente por volume ou por peso) do item são utilizadas para resumir sua importância em um contexto ecológico. Além das fontes de erro inerentes ao método escolhido, tais como diferentes taxas de digestão das presas, há inconvenientes quanto à obtenção dos dados de peso e volume de itens diminutos, tais como algas e detritos, que compõem parte significativa da dieta de muitas espécies de peixes.

Na busca de alternativas à quantificação por peso ou volume, Guillen \& Granado (1984) propuseram um método baseado em estimativas visuais, que refletem a área relativa ocupada por cada item. Outros autores apontam problemas no uso de estimativas visuais para aferir a importância de determinados itens e, tentando contornar o problema, propuseram outros índices e estratégias de análise menos subjetivas (Braga 1999, Lima-Júnior \& Goitein 2001). Apesar de ser visualmente orientada, a dominância não mostra tal subjetividade, pois reflete o número de vezes que o item se destaca no conjunto de indivíduos analisados.

Em razão de peso e dominância apresentarem valores muito semelhantes na presente análise, propõe-se que a dominância seja utilizada como alternativa à pesagem dos itens alimentares. Além de ser de aplicação simples, a dominância reflete a importância quantitativa dos itens mais expressivos na dieta e, por ser associada também à ocorrência, visto que é calculada em função do número total de exemplares analisados, gera valores muito similares ao IAi, conforme demonstrado por meio do índice de similaridade de Morisita-Horn. Observou-se ainda que a dominância não mostrou restrições quanto ao tamanho dos itens alimentares, que variaram desde pequenos a grandes organismos (p. ex., algas a peixes) ou de pequenas partículas a grandes fragmentos (p. ex., detritos a restos vegetais). Finalmente, quando associada à representação gráfica proposta por Costello (1990), a dominância também permite identificar as tendências de especialização/generalização alimentar de um conjunto de indivíduos.

Diante do que foi discutido acima, destaca-se nitidamente a importância da pergunta ecológica que deverá ser respondida. Se o objetivo do estudo for quantificar a importância de cada item alimentar, respondendo também se há tendências à especialização/generalização na dieta, a dominância representada no esquema gráfico proposto por
Costello (1990) substitui adequadamente a pesagem dos itens e o cálculo de índices compostos, com a vantagem de simplificar a etapa de coleta e análise de dados.

\section{Agradecimentos}

Agradecemos Katharina E. Esteves e Norma S. Hahn por valiosas sugestões, Oscar A. Shibatta pela identificação das espécies e curadoria dos exemplares utilizados, IBAMA pela permissão de coleta $\left(\mathrm{n}^{\circ}\right.$ 083/2003) e revisores anônimos pelas importantes sugestões. Este trabalho foi parcialmente subvencionado pelo Projeto "Caracterização biológica da ictiofauna do reservatório de Capivara UHE Escola Mackenzie”, convênio FAUEL/UEL/Duke Energy, e pela Fundação de Amparo à Pesquisa do Estado de São Paulo (FAPESP) dentro do Programa BIOTA/FAPESP (www.biota.org.br) (01/13340-7, 02/05996-2).

\section{Referências bibliográficas}

AMUNDSEN, P.A., GABLER, H.M. \& STALDVIK, F.J. 1996. A new approach to graphical analysis of feeding strategy from stomach contents data - modification of the Costello (1990) method. J. Fish Biol. 48:607-614.

ARANHA, J.M.R. 1993. Método para análise quantitativa de algas e outros itens microscópicos de alimentação de peixes. Acta Biol. Par. 22:71-76.

BENNEMANN, S.T., SHIBATTA, O.A. \& GARAVELLO, J.C. 2000. Peixes do Rio Tibagi: uma abordagem ecológica. Editora Universidade Estadual de Londrina, Londrina.

BENNEMANN, S.T., CAPRA, L.G., GALVES, W. \& SHIBATTA, O.A. 2006. Dinâmica trófica de Plagioscion squamosissimus (Perciformes, Sciaenidae) em trechos de influência da represa Capivara (rios Paranapanema e Tibagi). Iheringia, Sér. Zool.96:115-119.

BOWEN, S.H. 1992. Quantitative description of the diet. In Fisheries techniques (L.A. Nielsen \& D.L. Johnson, eds). American Fisheries Society, Bethesda, p.325-336.

BRAGA, F.M.S. 1999. O grau de preferência alimentar: um método qualitativo e quantitativo para o estudo do conteúdo estomacal de peixes. Acta Scientiarum 21:291-295.

CASATTI, L. 2003. Family Sciaenidae (Drums or croakers). In Check list of the freshwater fishes of South and Central America (R.E. Reis, S.O. Kullander \& C. J. Ferraris Jr., orgs). EDIPUCRS, Porto Alegre, p.599-602.

CASTRO, R.M.C. \& CASATTI, L. 1998. The fish fauna from a small forest stream of the upper Paraná River basin, southeastern Brazil. Ichthyol. Explor. Freshwaters 7:337-352.

COSTELLO, M.J. 1990. Predator feeding strategy and prey importance: a new graphical analysis. J. Fish Biol. 36:261-263. 
ESTEVES, K.E. \& GALETTI, P.M. 1994. Feeding ecology of Moenkhausia intermedia (Pisces, Characidae) in a small oxbow lake of Mogi-Guaçú River, São Paulo, Brazil. Verh. Internat. Verein. Limnol. 25:2198-2204.

FERRARIS, C.J. 2003. Subfamily Loricariinae (Armored catfishes). In Check list of the freshwater fishes of South and Central America (R.E. Reis, S.O. Kullander \& C. J. Ferraris Jr., orgs). EDIPUCRS, Porto Alegre, p.330-350.

FUGI, R., HAHN, N.S. \& AGOSTINHO, A.A. 1996. Feeding styles of five species of bottom-feeding fishes of the high Paraná River. Environ. Biol. Fishes 46:297-307.

GARAVELLO, J.C. \& BRITSKI, H.A. 2003. Family Anostomidae (Headstanders). In Check list of the freshwater fishes of South and Central America (R.E. Reis, S.O. Kullander \& C. J. Ferraris Jr., orgs). EDIPUCRS, Porto Alegre, p.71-84.

GUILLEN,E.\&GRANADO,C.1984.Alimentacióndelaictiofauna del embalse de Torrejon(rio Tajo, Caceres). Limnética 1:304-310.

HAHN, N.S. \& DELARIVA, L. 2003. Métodos para avaliação da alimentação natural de peixes: o que estamos usando? Interciencia 28:100-104.

HAHN, N.S., AGOSTINHO,A.A. \& GOITEIN, R. 1997. Feeding ecology of curvina Plagioscion squamossissimus (Hechel, 1840) (Osteichthyes, Perciformes) in the Itaipu Reservoir and Porto Rico floodplain. Acta Limn. Bras. 9:11-22.

HAHN, N.S., AGOSTINHO,A.A., GOMES, L.C. \& BINI, L.M. 1998. Estrutura trófica da ictiofauna do reservatório de Itaipu (Paraná-Brasil) nos primeiros de sua formação. Interciencia 23:299-305.

HYNES, H.B.N. 1950. The food of fresh-water sticklebacks (Gasterosteus aculeatus and Pygosteus pungitius), with a review of methods used in studies of the food of fishes. J. Anim. Ecol. 19:36-57.

HYSLOP, E.J. 1980. Stomach contents analysis - a review of methods and their application. J. Fish Biol. 17:411-429.

KAWAKAMI, E. \& VAZZOLER, G. 1980. Método gráfico e estimativa de índice alimentar aplicado no estudo de alimentação de peixes. Bol. Inst. Oceanogr. 29:205-207.

KREBS, C.J. 1999. Ecological methodology. Addison Wesley Longman, New York.

LIMA, F.C.T. et al. 2003. Genera incertae sedis in Characidae. In Check list of the freshwater fishes of South and Central America (R.E. Reis, S.O. Kullander \& C. J. Ferraris Jr., orgs). EDIPUCRS, Porto Alegre, p.106-169.

LIMA-JÚNIOR, S.E. \& GOITEIN, R. 2001. Anew method for the analysis of fish stomach contents. Acta Scientiarum 23:421-424.

LUCINDA, P.H.F. 2003. Family Poeciliidae (Livebearers). In Check list of the freshwater fishes of South and Central America (R.E. Reis, S.O. Kullander \& C. J. Ferraris Jr., orgs). EDIPUCRS, Porto Alegre, p. 555-581.

OLIVEIRA, A.K., ALVIM, M.C.C., PERET, A.C. \& ALVES, C.B.M. 2004. Diet shifts related to body size of the pirambeba Serrasalmus brandtii Lütken, 1875 (Osteichthyes, Serrasalminae) in the Cajuru Reservoir, São Francisco River basin, Brazil. Braz. J. Biol. 64:117-124.
OLIVEIRA, D.C. \& BENNEMANN, S.T. 2005. Ictiofauna, recursos alimentares e relações com as interferências antrópicas em um riacho urbano no sul do Brasil. Biota Neotropica 5(1): http://www.biotaneotropica.org.br/ v5n1/pt/download?article+BN02905012005

PINKAS, L., OLIPHANT, M.S. \& IVERSON, I.L.K. 1971. Food habits of albacore, bluefin tuna and bonito in Californian waters. Calif. Fish Game 152:1-105.

ROSECCHI, E. \& NOUAZE, Y. 1987. Comparison de cinq indices alimentaires utilisés dans l'analyse des contenus stomacaux. Rev. Trav. Inst. Pêches marit. 49:111-123.

SABINO, J. 1999. Comportamento de peixes em riachos: métodos de estudo para uma abordagem naturalística. In Ecologia de peixes de riachos: estado atual e perspectivas (E.P. Caramaschi, R. Mazzoni, C.R.S.F. Bizerril, P.R. Peres-Neto, eds). Oecologia Brasiliensis, v. VI, PPGE-UFRJ, Rio de Janeiro, p.183-208.

SCHAEFER, S.A. 1998. Conflict and resolution: impact of new taxa on phylogenetic studies of neotropical cascudinhos (Siluroidei: Loricariidae). In Phylogeny and classification of neotropical fishes (L.R., Malabarba, R. E. Reis, R. P. Vari, Z. M. S. Lucena \& C. A. S. Lucena, eds). EDIPUCRS, Porto Alegre, p.375-400.

UIEDA, V.S. \& CASTRO, R.M.C. 1999. Coleta e fixação de peixes de riachos. In Ecologia de peixes de riachos: estado atual e perspectivas (E.P. Caramaschi, R. Mazzoni, C.R.S.F. Bizerril, P.R. Peres-Neto, eds). Oecologia Brasiliensis, v. VI, PPGE-UFRJ, Rio de Janeiro, p.1-22.

VITULE, J.R.S. \& ARANHA, J.M.R. 2002. Ecologia alimentar do lambari, Deuterodon langei Travassos, 1957 (Characidae, Tetragonopterinae), de diferentes tamanhos em um riacho da Floresta Atlântica, Paraná (Brasil). Acta Biol. Par. 31:137-150.

WINDELL, J.T. \& BOWEN, S.H. 1978. Methods for study of fish diets based on analysis of stomach contents. In Methods for assessment of fish production in fresh waters (T. Bagenal, ed.), Blackwell Scientific, Oxford, p.219-223.

Título: Alimentação de peixes: proposta para análise de itens registrados em conteúdos gástricos

Autores: Sirlei Terezinha Bennemann, Lilian Casatti \& Deise Cristiane de Oliveira

Biota Neotropica, Vol. 6 ( número 2): 2006

http://www.biotaneotropica.org.br/v6n2/pt/ abstract?article+bn01206022006

Recebido em 27/07/05 - Revisado em 08/04/06 Publicado em 01/05/06.

ISSN 1676-0603 